\title{
Vitaminas e antioxidantes na degeneração macular relacionada à idade
}

\author{
Vitamins and antioxidants in age-related maculardegeneration
}

PedroDurães Serracarbassa ${ }^{1}$

${ }^{1}$ Doutor em Medicina pela Faculdade de Medicina da Universidade de São Paulo - USP - São Paulo (SP) - Brasil. Médico colaborador do Departamento de Oftalmologia do Hospital das Clinicas - Faculdade de Medicina da Universidade de São Paulo - USP - São Paulo. Brasil.

Endereço para correspondência: Pedro Durães Serracarbassa - Av. Brig. Faria Lima 1903, cj. 43 - São Paulo - SP - CEP 01452-001

E-mail: serracar@brfree.com.br

Recebido para publicação em 26.09.2005 Aprovação em 13.12.2005

Nota Editorial: Depois de concluída a análise do artigo sob sigilo editorial e com a anuência do Dr. Walter Yukihiko Takahashi sobre a divulgação de seu nome como revisor, agradecemos sua participação neste processo.

\begin{tabular}{|l|}
\hline RESUMO \\
\hline O autor descreve os efeitos bioquímicos e estruturais das vitaminas e \\
antioxidantes na retina. Apresenta as principais substâncias presentes na \\
dieta alimentar e na suplementação vitamínica envolvidas na gênese da \\
degeneração macular relacionada à idade. Relata ainda os resultados de \\
estudos prospectivos multicêntricos relacionados ao assunto, por meio de \\
revisão bibliográfica.
\end{tabular}

Descritores: Degeneração macular/prevenção \& controle; Antioxidantes/administração \& dosagem; Antioxidantes/uso terapêutico; Suplementos dietéticos; Zinco/administração \& dosagem; Cobre/administração \& dosagem; Dieta

\section{INTRODUÇÃO}

As reações fotoquímicas originadas na retina e no epitélio pigmentado da retina tornam estas estruturas altamente susceptíveis aos danos causados pelo estresse oxidativo. O processo de oxidação gera moléculas instáveis com um número ímpar de elétrons, chamados radicais livres. Para atingir a estabilidade de elétrons, estas moléculas reagem com os tecidos oculares subjacentes ${ }^{(1)}$.

Os mecanismos de defesa destas camadas do globo ocular incluem a presença das substâncias antioxidantes. Alguns antioxidantes são produzidos pelo próprio organismo e outros, como o ácido ascórbico, $\alpha$-tocoferol e os carotenóides luteína-zeaxantina, são ingeridos ${ }^{(2)}$.

A reposição destas substâncias antioxidantes pode ser realizada na forma de suplementos vitamínicos ou pela dieta alimentar. O quadro a seguir mostra os principais vegetais e frutas como maior poder oxidante, segundo o Departamento de Agricultura dos Estados Unidos (USDA) ${ }^{(3)}$.

Estudos mostram que a suplementação com alimentos ricos em carotenóides (especialmente a luteína e a zeaxantina) tem a capacidade de aumentar a concentração e a densidade do pigmento macular ${ }^{(4)}$. Estes dois compostos da família dos carotenóides são encontrados em níveis aproximadamente $50 \%$ maiores em olhos normais do que em olhos portadores de degeneração macular relacionada à idade $(\mathrm{DMRI})^{(5)}$. A luteína e zeaxantina concentram-se nos axônios dos fotorreceptores e interneurônios da camada plexiforme interna da mácula. Os possíveis efeitos protetores são: absorção dos comprimentos de onda associados ao dano fotoquímico da retina sensorial e remoção dos radicais livres e formas reativas de oxigênio geradas pela atividade metabólica ${ }^{(6)}$. Outras substâncias envolvidas na patogênese da DMRI seriam os ácidos graxos poli-insaturados de cadeia longa omega-3 (LCP-UFA) ingeridos na dieta lipídica ou encontrado nos peixes ${ }^{(7)}$. 


\begin{tabular}{|cll|}
\hline \multicolumn{3}{c}{ Quadro. Poder antioxidante } \\
\hline 1 & Vegetais & Frutas \\
2 & Repolho & Morango \\
3 & Beterraba & Ameixa \\
4 & Pimenta vermelha & Laranja \\
5 & Brócolis & Uva \\
6 & Espinafre & Maçã \\
7 & Batata & Tomate \\
8 & Milho & Banana \\
9 & - & Pêra \\
\hline
\end{tabular}

Apesar dos estudos citados, os benefícios anatômicos e funcionais da suplementação vitamínica ou dieta alimentar na DMRI permanecem controversos. O fato se deve ao pequeno número de trabalhos prospectivos com grupo-controle e aos dados inconsistentes sugerindo possíveis efeitos protetores dos antioxidantes.

Alguns estudos prospectivos têm examinado a relação entre a suplementação com antioxidantes e a DMRI. O "Beaver Dam Eye Study" demonstrou uma discreta associação entre a ingestão de zinco e uma diminuição do risco de aparecimento da DMRI precoce em cinco anos de acompanhamento ${ }^{(8)}$. Em outro trabalho prospectivo, a análise dos dados do "Nurses Health Study" e do "Health Professionals Follow-up Study" não mostrou qualquer efeito protetor para a DMRI, nos indivíduos que ingeriram doses moderadas de zinco na sua dieta regular ou na forma de suplemento em 8 anos. As doses de zinco ingeridas nestes estudos variaram de 8,5 a $25,5 \mathrm{mg} / \mathrm{dia}$ nas mulheres e 9,9 a 40,1 mg/dia nos homens ${ }^{(9)}$.

Outro estudo prospectivo, o "Blue Montains Eye Study", não encontrou uma associação significativa entre a ingestão de antioxidantes e a incidência de DMRI precoce ${ }^{(10)}$. A população estudada foi questionada quanto à presença, na sua dieta regular ou como suplemento, dos cinco carotenóides ( $\alpha$-caroteno, $\beta$-caroteno, $\beta$-criptoxantina, luteína-zeaxantina e licopeno), dos antioxidantes vitamina $\mathrm{C}$ e zinco, além do retinol e vitamina $\mathrm{A}$. Porém, as doses ingeridas foram inferiores ao "Age-Related Eye Disease Study" (AREDS), como citado a seguir ${ }^{(11)}$.

O AREDS foi um estudo multicêntrico, duplo-mascarado, randomizado, que envolveu pacientes com mais de 55 anos de idade, não-fumantes (devido à associação de altas doses de $\beta$ caroteno com câncer de pulmão em fumantes), que apresentavam as seguintes características abaixo:

- Grupo 1: Ausência de DMRI, poucas ou pequenas drusas

- Grupo 2: DMRI leve, muitas drusas pequenas ou poucas drusas médias em um ou ambos os olhos, ou anormalidades do pigmento macular

- Grupo 3: DMRI moderada, muitas drusas médias ou uma ou mais drusas grandes em um ou ambos os olhos

- Grupo 4: DMRI avançada, atrofia do epitélio pigmentado da retina ou membrana neovascular de coróide com baixa visão em um olho.
Os participantes foram alocados em grupos e receberam cápsulas diárias, por via oral, contendo:

- Grupo 1: antioxidantes

- Grupo 2: zinco e cobre

- Grupo 3: antioxidantes e zinco

- Grupo 4: placebo

Os resultados da fase II do estudo mostraram que os pacientes com drusas pequenas (Grupo 2) tinham um risco muito baixo $(1,3 \%)$ de desenvolverem a forma avançada da DMRI em cinco anos. Por esta razão, apenas os indivíduos portadores das formas intermediária e avançada da DMRI (Grupo 3 e 4) foram analisados.

A conclusão do estudo mostrou que a combinação dos antioxidantes: Vitamina C $500 \mathrm{mg}$, Vitamina E 400UI, Betacaroteno $15 \mathrm{mg}$ (ou Vitamina A $25000 \mathrm{UI}$ ) mais o óxido de zinco $80 \mathrm{mg}$ e óxido cúprico $2 \mathrm{mg}$, diminuiu o risco de progressão para a forma avançada da DMRI em $25 \%$ e reduziu o risco de diminuição da visão em $27 \%$.

Outras conclusões ainda preliminares mostraram que a ingestão de omega-3 LCP-UFA na dieta regular ou encontrado nos peixes diminuiu o risco de progressão para a forma neovascular da DMRI ${ }^{(12)}$.

Portanto, recomenda-se que pacientes com mais de 55 anos, não-fumantes, portadores de formas intermediárias ou avançadas da DMRI, sejam tratados com suplementações vitamínicas baseadas no AREDS. Doses diferentes daquelas recomendadas pelo AREDS, ou dietas alimentares ricas em carotenóides, carecem de comprovação científica até o presente momento.

\section{ABSTRACT}

The author describes biochemical and structural effects of vitamins and antioxidants on the retina. The main substances present in diet food and vitamin supplies involved in the genesis of age-related macular degeneration are shown. Also reports on the outcomes of prospective studies related to the subject, by literature review are presented.

Keywords: Macular degeneration/prevention \& control; Antioxidants/administration \& dosage; Antioxidants/therapeutic use; Dietary supplements, Zinc/administration \& dosage; Copper/administration \& dosage; Diet

\section{REFERÊNCIAS}

1. Ham WT Jr, Mueller HA, Sliney DH. Retinal sensitivity to damage for short wavelenght light. Nature. 1976:260(5547):153-5

2. Farnsworth CC, Dratz EA. Oxidative damage of retinal rod outer segment menbranes and the role of vitamin E. Biochem Biophys Acta. 1976;443(3):556-70.

3. Chung-Ahuja JK, Holden JM, Forman MR, Mangels AR, Beecher GR, Lanza E. The development and application of carotenoid database for fruits, vegetables, and selected multicomponent foods. J Am Diet Assoc. 1993;93(3):318-23.

4. Hammond BR Jr, Johnson EJ, Russell RM, Krinsky NI, Yeum KJ, Edwards $\mathrm{RB}$, et al. Dietary modification of human macular pigment density. Invest Ophthalmol Vis Sci. 1997;38(9):1795-801. 
5. Landrum JT, Bone RA, Kilburn MD. The macular pigment: a possible role in protection from age-related macular degeneration. Adv Pharmacol. 1997;38: 537-56.

6. Landrum JT, Bone RA, Joa H, Kilburn MD, Moore LL, Sprague KE. A one year study of the macular pigment: the effect of 140 days of a lutein supplement. Exp Eye Res. 1997;65(1):57-62.

7. Smith W, Mitchell P, Leeder SR. Dietary fat and fish intake and age-related maculopathy. Arch Ophthalmol. 2000;118(3):401-4.

8. VandenLangenberg GM, Mares-Perlman JA, Klein R, Klein BE, Bradi WE, Palta M. Associations between antioxidant and zinc intake and the 5-year incidence of early age-related maculopathy in the Beaver Dam Eye Study. Am J Epidemiol. 1998;148(2):204-14.

9. Cho E, Stampfer MJ, Seddon JM, Hung S, Spiegelman D, Rimm EB, et al. Prospective study of zinc intake and the risk of age-related macular degeneration. Ann Epidemiol. 2001;11(5):328-36.
10. Smith W, Mitchell P, Webb K, Leeder SR. Dietary antioxidants and agerelated maculopathy. The Blue Mountains Eye Study. Ophthalmology. 1999; 106(4):761-7.

11. Aged-Related Eye Disease Study Research Group. A randomized, placebocontrolled, clinical trial of high-dose supplementation with vitamins $\mathrm{C}$ and $\mathrm{E}$, beta carotene, and zinc for age-related macular degeneration and visual loss. AREDS report n.8. Arch Ophthalmol. 2001;119(10):1417-36. Comment in: Arch Ophthalmol. 2001;119(10):1533-4; Arch Ophthalmol. 2002;120(1):100-1; Arch Opthalmol. 2002;120(7):997; author reply 997-9; Arch Ophthalmol. 2002;120(11):1602; Arch Ophthalmol. 2003;121(3):416-7; J Fam Pract. 2002;51(2):105; JAMA. 2001;286(19):2466-8.

12. Cho E, Hung S, Willett WC, Spiegelman D, Rimm EB, Seddon JM, et al. Prospective study of dietary fat and the risk of age-related macular degeneration. Am J Clin Nutr. 2001;73(2):209-18.

\section{$56^{\circ}$ Jornada do Centro Brasileiro de Estrabismo}

\section{1 de Outubro de 2006 Jurerê Beach Village Florianópolis - SC}

\section{INFORMAÇÕES}

Toll free: $0800-48110$ E-mail: reservahoteis@jurere.com.br•Home page: www.cbe.org.br 